\title{
Scanned Probe-Based Chemical and Thermomechanical Property Mapping of Polymeric Materials Suitable for Electronic Applications
}

\author{
L.T. Germinario, ${ }^{*}$ K. Kjoller, ${ }^{* *}$ C.B. Prater, ${ }^{* *}$ and K. Sahagian, ${ }^{* *}$ \\ * Department of Physics and Astronomy, Appalachian State University, Boone, NC \\ ** Anasys Instruments, Santa Barbara, CA
}

The emergence of polymers in consumer electronics stems from their ease of processing, low production cost, ability to flex and their tunable properties at the molecular level. This has led to an increased demand for innovation in materials research that inherently requires small-scale testing due to the micro and nano-scale feature sizes of components. At these dimensions, spatial confinement and interfacial effects can lead to significant deviations in mechanical and thermal properties from the bulk. Additionally, since processing is often performed below the polymer's glass transition $\left(\mathrm{T}_{\mathrm{g}}\right)$, the initial morphology developed during manufacturing is unstable as it is kinetically trapped in a non-equilibrium state. This leads to a tendency for crystallization, large scale phase segregation of the nanostructure and subsequent degradation of properties (1).

To advance our understanding of length-scale and processing effects on material properties, a scanned probe-based approach (nanoIR, Anasys Instruments) is described that combines near-field probing of vibrational resonances of molecules excited by a tunable Infrared (IR) laser [2] with the nanoscale resolution of atomic force microscopy (AFM). MEMS-based silicon microcantelevers with integrated solid state heaters [3] can also be used to combine thermal property data supplied by localized heating with a thermal probe, with the chemical specificity of vibrational spectroscopy; all on an AFM platform and at nanometer length-scales.

To detect and analyze temperature-time-dependent transitions in organic and polymeric materials at high spatial resolution, new techniques that employ sharp, heatable probes have been developed that enable both localized thermal analysis (LTA) as well as provide mapping and visualization of thermal transitions at the micro and nanoscale [4]. In addition, the chemical information that was previously lacking in AFM is gained by employing a tunable, pulsed IR laser that uses the AFM cantilever deflection system to detect the rapid thermal expansion of the sample due to localized heating by the absorbed IR radiation. This photothermal induced expansion produces a resonance (PTIR) and ringdown that is recorded as a function of time and is converted electronically to a frequency spectrum that provides both chemical sensitivity and contact stiffness information at $\sim 100$ $\mathrm{nm}$ length-scale [2]. The laser linewidth is generally $<10 \mathrm{~cm}^{-1}$ over a range from $1200-3600 \mathrm{~cm}^{-1}$.

Examples demonstrate nanoscale ordering of electroactive, ionic liquid-containing (IL), zwitterionic copolymers (5) of both dry and swollen with the ionic liquid, 1-ethyl-3-methylimidazolium ethyl sulfate (EMIm ES) (figure 1A). The appearance of low $T_{g}$ peaks in dry and EMIm ES swollen films was detected after repeated LTA measurements from fixed points. These results suggest the development of a thermally-induced nanoscale, phase-separated structure. The measured morphology and swelling behavior is also in agreement with Molecular Dynamics models established for perfluorinated ionomer membranes (6). 
Organic photovoltaic blends consisting of poly(3-hexylthiophene) (P3HT) and PCBM ([6'6]-phenyl C61-butyric acid methyl ester were also characterized by AFM and spectral data were recorded from select points (figure 1B). Spatially resolved spectra provide direct chemical evidence for a phase separation of active layer components by the appearance of a splitting of the methylene peak at $1444 \mathrm{~cm}^{-1}$ and $1432 \mathrm{~cm}^{-1}$ and by a corresponding increase in carbonyl stretch band at $\sim 1730 \mathrm{~cm}^{-1}$, associated with PCBM. Spectroscopic differences could also be detected from regions that were previously heated by thermal probes and offered direct evidence of crystallization and phase transformation of nanostructures that develop as a result of localized heating.

Multiple heating rates were also performed during LTA experiments to detect and identify kinetic processes such as evaporation, crystallization, crosslinking, or decomposition. A shift in the measured transition temperature is indicative of a kinetic process (7).

Acknowledgement. The authors wish to thank Drs. Timothy E. Long (Virginia Tech.) and Rebecca H. Brown (ORNL) for providing zwitterionic copolymers. This research was partially supported by the ASU, AppNano Microscopy Laboratory through the National Science Foundation Award MRI0821124 .

References:

[1] P. Vanlaeke, G. Vanhoyland G, T. Aernouts, et al. Thin Solid Films, 511 (2006) 358.

[2] K. Kjoller, J.R. Felts, D. Cook, et al. Nanotechnology, 21, 185705 (2010).

[3] B. A. Nelson and W.P. King, Rev. Sci, Instrum., 78, 023702 (2007).

[4] L.T. Germinario, C.K. Schoff, K. Kjoller, et al., JCT Coatings Tech 7 (2) (2010) 36.

[5] R. H. Brown, A. J. Duncan, J-H. Choi, et al., Macromolecules, 43 (2010) 790.

[6] K.A. Mauritz, and R.B. Moore, Chemical Reviews, 104 (2004) 4535.

[7] G.S. Grest and M.H. Cohen, Phys. Rev., 21 (9) (1980) 4113.

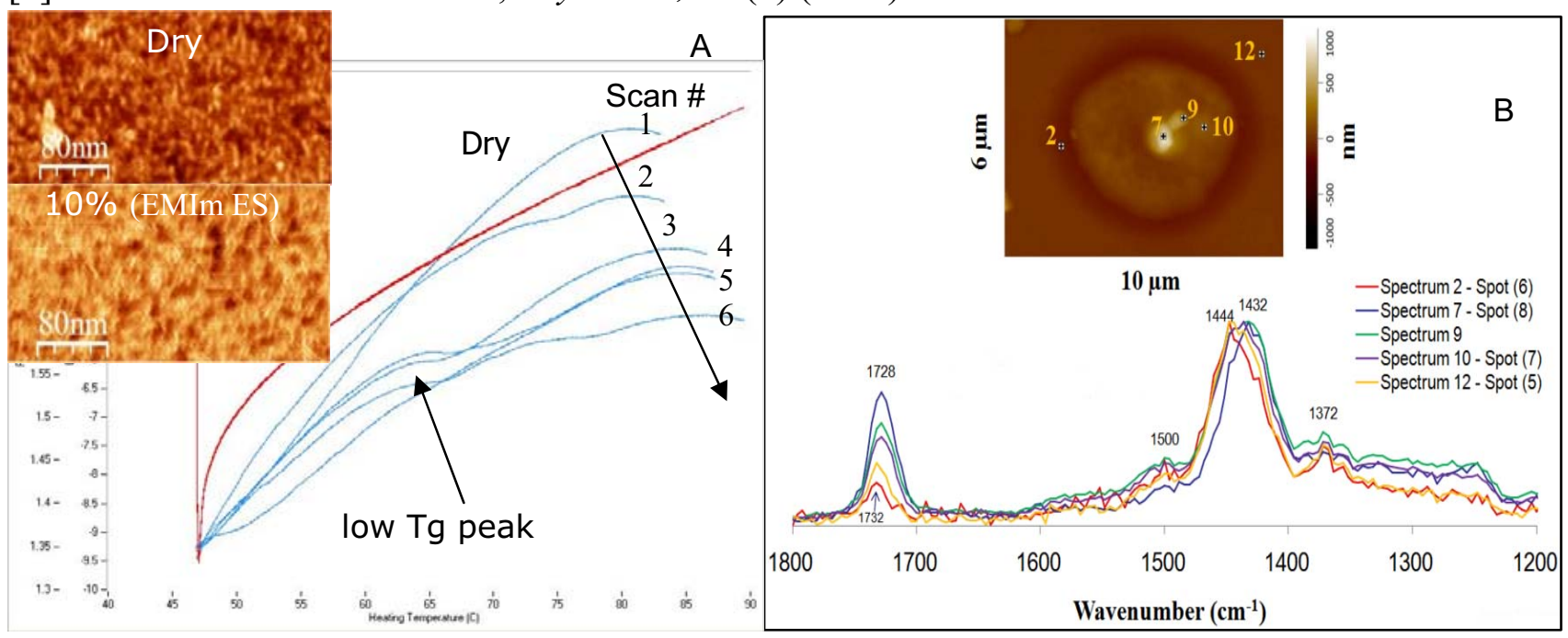

FIG 1. (A) Phase imaging of zwitterionic copolymers show an ordering at the nanoscale. Repeated LTA from fixed points on surfaces of dry, 5 and 10\% IL show the appearance of a lower $\mathrm{T}_{\mathrm{g}}$ peak possibly due to phase separation and migration. (B) AFM topography of an organic photovoltaic blend, (P3HT/ PCBM) and corresponding point spectra from marked regions provide direct evidence for a spatially varying chemical composition as evidenced by a splitting of the methylene peak at $1444 \mathrm{~cm}^{-1}$ and $1432 \mathrm{~cm}^{-1}$ and by a corresponding increase in carbonyl stretch band at $\sim 1730 \mathrm{~cm}^{-1}$. 